\title{
AUTOPARK: A Sensor Based, Automated, Secure and Efficient Parking Guidance System
}

\author{
${ }^{1}$ Surbhi Maggo, ${ }^{2}$ Reema Aswani \\ ${ }^{1,2}$ Jaypee Institute of Information Technology, India
}

\begin{abstract}
Nowadays, due to modern and lavish lifestyles people prefer their own vehicles while going anywhere, resulting in increased traffic congestion and parking problems. In this paper, we propose and present a completely automated system AUTOPARK that will solve the parking issues without any human intervention. The system will comprise of an infrastructure based wired sensor network with sensors placed at each parking slot to detect the occupancy of the parking slots. It also comprises of an automated voice enabled system for guiding vehicles to the available vacant slots by giving directions. The system also enables security and takes into consideration the prevention of theft of vehicles, for this purpose we incorporate face detection and number plate recognition modules so that a track record can be maintained. In case of any mishap or unidentified car or person, communication can be made with base station for immediate human assistance. The paper further discusses design model, the architectural layout and methodology used to build AUTOPARK. It concludes with system evaluation and efficiency analysis of the system.
\end{abstract}

Keywords - Face Detection, Number plate recognition, Pressure sensor, Text-to-Speech, Ultrasonic Sensor

\section{INTRODUCTION}

The urban population in India has increased significantly from 62 million in 1951 to 285 million in 2001 and is estimated to grow to around 540 million by the year 2021. In terms of percentage of total population, the urban population has gone up from 17\% in 1951 to $29 \%$ in 2001 and is expected to increase up to around $37 \%$ by the year 2021 . Consequently, the number and size of cities have also increased considerably [1].

All most all large metropolitan cities face the same problem that the public transport system is either obsolete or is insufficient to cater to the commuter demands and needs. As a result of which, public transportation is associated with unfavorable consequences like discomfort due to overcrowding, time delays due to long routes and unsatisfactory service due to lack of maintenance. These problems and constraints force the people to travel by their own cars.

According to Motor Transport Statistics [1]-[2], the annual rate of growth of motor vehicles in India was around 11 percent during the last decade. In 1987 there were 12.6 million vehicles. 10 years later, in 1997, this number had increased threefold to 37.2 million. Vehicle population is estimated to be over 50 million by 2001. The basic problem is not the number of vehicles in the country but their over-concentration in a few selected cities, particularly in metropolitan cities. Unfortunately the metropolitan cities lack adequate parking space to accommodate this huge inflow of vehicles.

Moreover, people have adopted a modern and a fast paced lifestyle where owning a car is a must. With increasing modern facilities and malls, people visit the malls very frequently, even for day to day grocery shopping. Looking at the growing number of vehicles on Indian roads and the inadequate capacity to accommodate them, there is a need for an efficient car parking management system. For this reason, research on automation in the field of parking management came into being. Automation in this parking industry is a boon, as it saves time, helps in optimal and efficient use of space and saves manpower unlike the conventional parking system.

Keeping in mind all these problems faced in metropolitan cities, AUTOPARK is designed to solve the problem of traffic congestion and efficient parking in malls and multiplexes, by automating this process to make it faster, more reliable cost-effective and secure. It ensures that the car enters the parking area only if there is a vacant slot available, thus reducing delays and unnecessary inconvenience to the people while parking. The system also provides voice enabled direction guidance to the user on entry to the parking with security ensured by keeping a track of the number plate and image of the person by number plate recognition and face detection respectively.

The main objectives of any automated car parking system are: (a) Identifying availability of vacant slots; (b) Providing informative guidance to the user to conveniently reach the destined parking slot. (c) Providing security by preventing cars from being stolen. AUTOPARK covers all the above requirements and ensures a secure and autonomous toll free parking system. 
Section 2 discusses the related literature survey with a description of the existing parking management systems. Section 3 gives an overview of the entire system with system requirements. Further section 4 depicts the detailed implementation of the system with different modules for entry and exit operations. The section also highlights the main features with their detailed description that distinguish AUTOPARK from already existing parking management systems. Section 5 discusses the results of the proposed system supported with suitable statistics. The paper concludes with the system evaluation in Section 6 which presents the efficiency and reliability of the system and conclusion and future work in Section 7 and 8 respectively.

\section{RELATED WORK}

This section reviews the already existing automated parking management systems. A general problem faced in all such systems is vehicle identification and tracking.

A similar system "Zigbee and GSM Based Secure Vehicle Parking Management and Reservation System" proposed by Aswin Sayeeraman and P.S.Ramesh [3] also uses Zigbee Technology along with GSM for parking management. This system comprises of three main modules namely (a) Checking for availability of free parking slot; Slot booking (c) Password enabled security system after the car is parked in the reserved slot. The user can book the slot incase free slots are available via SMS and a password is given to each user. On entering the password at the entry gate the user is able to park the car in a secure way. The system ensures security and reservation of slot but doesn't provide any guidance to reach the designated slot. In addition, each slot is equipped with a Zigbee Module thus increasing the cost overhead.

A hybrid approach for an intelligent parking system was proposed by Lee, Yoon and Ghosh [4] which uses a combination of ultrasonic and magnetic sensors. They used real world examples to show that their results were accurate and practical. Though it provided a cheap and accurate solution, the main aim was to get the count of number of vehicles on different floors. The scope of the work was restricted to vehicle detection rather than providing a smarter solution for the parking issues and congestion problems.

Another similar work in the area of parking system automation and management was an optical WSN (wireless sensor network) as a vehicle counting system in smart parking garages, proposed by Jatuporn et al. [5]. The solution proposed by them was to use an optical sensor head additionally with the wireless sensor node. The two sensor heads classified the objects of dissimilar sizes hence differentiating and identifying different sized vehicles. Yet again the solution was not a complete solution towards parking management issues.

D-Systems Project [6] presented various issues for a reliable WSN system using magnetic sensors. In this project a car park management system was implemented using a tiered architecture. The architecture is detailed using magnetic sensor boards. Various recommendations on the use of dynamic and robust routing, delayed retransmission etc was also proposed.

Iris-Net [7] proposed a wide-area sensor network architecture using motion detectors, video cameras and microphones. Web application was developed for clients from where they could acquire real time parking information which was generated using the sensor devices that detected free parking spaces. However the system generated huge amount of data leading to high power scavenging and communication bandwidth which are the major limitations of any wireless sensor network.

\section{SYSTEM MODEL AND ARCHITECTURE}

Keeping in mind the increased traffic congestion and the fast pace lifestyle, AUTOPARK is designed to reduce the effort and time required in conventional parking systems by automating the process of parking guidance. This section states the hardware and software requirements of the system along with the system architecture. The AUTOPARK has infrastructure based sensor network architecture and comprises of different modules that interact with each other to ensure a secure and a completely automated system for car parking in India. This parking system is a free of cost parking management system and can be used in any mall, multiplex or local market parking to ease the process of car parking.

System Architecture - Fig. 1 presents the system architecture of the Modeled Automated Parking Guidance System. It is an infrastructure based sensor and actuator network system where each of the parking slots in the parking area (as shown in Fig. 2) acts as a different node. Ultrasonic Distance Sensors are deployed at each node. These sensors are connected to the controllers (Arduino Duemilanove) at the entry gate through wired connections. Multiple sensor nodes are connected to a single Arduino. 


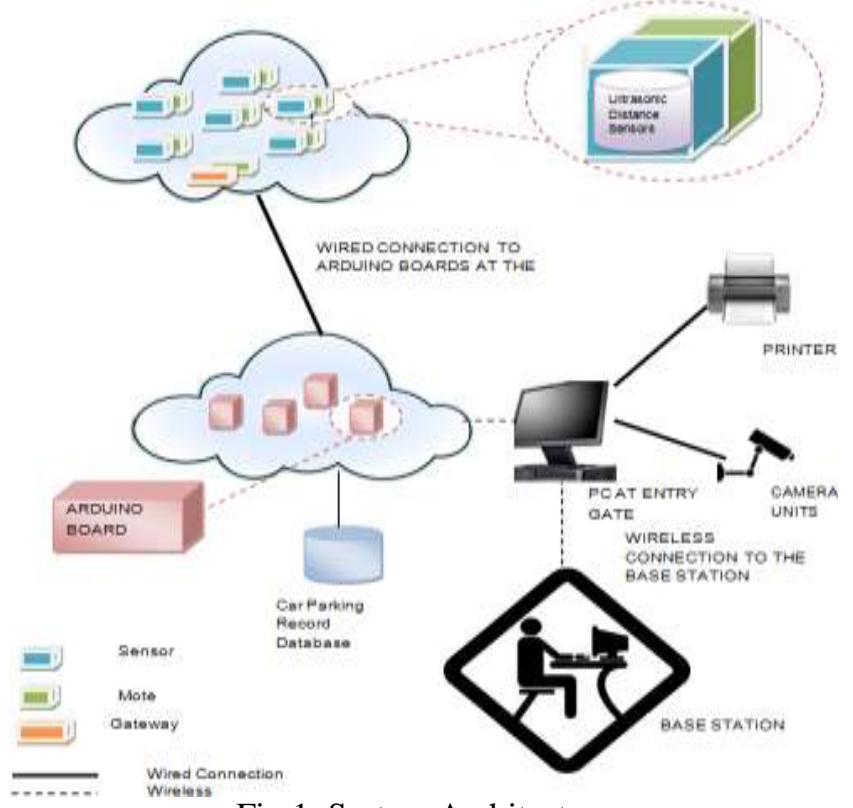

Fig 1: System Architecture

The Arduino boards are connected to the system at the entry gate. There are four camera units in the system two at the entry gate and two at exit, the cameras at the entry and exit gates are angled specifically to capture images of the driver's face and vehicles number plate at arrival and exit of the vehicles and send them to the system at the entry gate for processing via wired connection. The system at the gate is also connected to a base station to which it sends constant data/report for monitoring. This base station is under human surveillance and provides for human interference when required at the time of mismatch at exit gate. A speaker and a printer are also connected in wired fashion to the system at entry gate that provide for directions to the user towards the parking slot.

A specific region is marked at the entry as well as the exit where the vehicles are required to stand for their images to be captured. These demarcated regions are deployed with pressure sensors that detect the presence of the vehicle in the demarcated area and actuate by activating the cameras to take the images. The Ultrasonic sensors at the nodes not only help update the database of vacant/occupied slots but also at the time of exit from parking as they send signals of a slot being freed, actuation is performed via the controllers and LEDs from that slot till the exit are lighted to guide the vehicles towards the exit gate of the parking.

System Model - A complete view of the parking area developed as a prototype to test the designed automated Parking Guidance System is presented in Fig 2. System Requirements - A number of hardware and software requirements along with communication requirements between different hardware and software components for the construction of the Infrastructure based Sensor and Actuator Network for the automation of the parking guidance system are presented as follows. The system comprises of Arduino Duemilanove controller [8] which has an Atmega AT168 microcontroller that has a pre-installed boot loader, so one can download code to the board using only a USB-serial connection. The code written in Arduino Software can be uploaded to the microcontroller using the A-B USB cable.

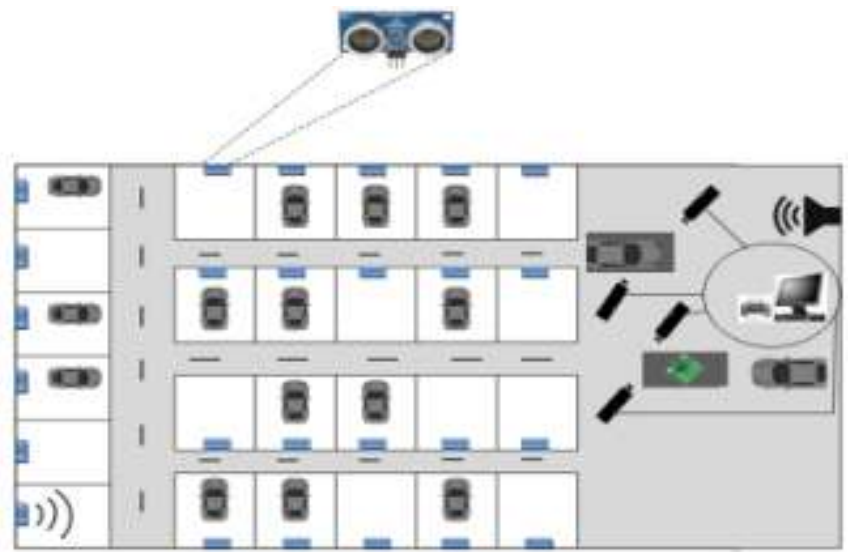

Fig 2: System Model of AutoPark 
Each slot is equipped with an Ultrasonic Distance Sensor [11] which is used to detect the occupancy of slots i.e. whether a particular slot is vacant or occupied. The connection between the sensor and the microcontrollers at the entry gate is wired, as the system follows an infrastructure based architecture and we are aware of the entire region of interest. Thus, a wired connection would be appropriate, reliable and cost-effective.

The ultrasonic sensor deployed at the slots provides precise distance measurements up to $3 \mathrm{~m}$. The sensor works by transmitting an ultrasonic burst and providing an output pulse that corresponds to the time required for the burst echo to return to the sensor. The distance to target can easily be calculated by measuring the pulse width. In this scenario, the target is the car being parked at each slot, and the distance is measured and occupancy is decided on the basis of a predefined threshold value.

The sensor values are aggregated at the entry gate where the microcontrollers are placed. The microcontrollers then transmit the data to the base station using serial communication. For experimentation purpose we have taken only one Arduino board attached with two sensors. Figure 1 shows the overview of the system.

Pressure sensors are also used in the system at the entry and exit gates. These pressure sensors are connected to the analog pin to measure the voltage output of the sensor. These sensors are deployed at the demarcated regions at the entry and exit to detect the presence of the vehicle. Here the pressure sensor MPXV5050gp is used to determine the presence of a vehicle. MPX5050GP piezoresistive transducer is a monolithic silicon pressure sensor (Figure) that combines advanced micromachining techniques, thin-film metallization, and bipolar processing to provide an accurate, high level analogue output signal that is proportional to the applied pressure. The MPX5050/MPXV5050G series pressure sensor [12]-[13] operating characteristics and internal reliability and qualification tests are based on use of dry air as the pressure media.

The sensor codes for the ultrasonic and pressure sensor are written in the Arduino software and are written on the Atmega chip on the Arduino board. The system also comprises of four cameras to ensure security at the entry and exit gate. All the cameras are connected to a computer (a sink node) that gathers data from the cameras as well as the parking slots. The entry gate has two cameras, one focused for capturing the image of the face of person and the second one for number plate identification. The images are stored in the database for comparison at the time of exit.

Similarly, at the time of exit, the image of the person and the number plate of the vehicle is again captured and compared with the already existing images in the database. This way the system ensures security and prevents theft of vehicles. There is a base station in the vicinity with a human assistant for help and is contacted in case of any mismatch while exiting the parking lot so that immediate human assistance can be provided to avoid mishaps or delays.

LEDs are placed on the floor of the parking area to direct all exiting cars by glowing the LEDs from the source of the exiting car to the destination i.e. the exit gate. The vehicle can simply follow the attached LEDs to reach the exit gate without any delay and inconvenience. A speaker connected at the entry gate gives directions to all entering vehicles using the directions generated by the system to the destined free slot via text to speech synthetic voice. Along with the voice enabled directions, a printed receipt with direction guidance and slot number is also generated automatically using a printer.

\section{IV.}

\section{SYSTEM IMPLEMENTATION}

\subsection{Functional modules of the system}

The system comprises of three main modules which can be classified as:

Module 1: Incoming Vehicle - This is further sub-divided into three modules:

a). Image Processing - In this module as the vehicle reaches the Entry gate, cameras capture images which are processed for drivers' face and number plate detection. The detected images are stored in the database to check at the time of exit in order to prevent incidents of theft etc.

b). Parking Guidance - In this module a check would be performed to look for vacant slots in the parking area. In case of 'No vacant slots', the gates are not opened for the vehicle and an apology message is displayed. In the presence of a vacant slot, directions from the entry gate till the free slot are generated by the system. These directions are provided to the user (vehicle) via synthetic voice using text to speech conversion for the directions.

c). Sensor Automation - In this module as the vehicle reaches the free slot and is getting parked the Ultrasonic Distance Sensor starts sensing the distance after a minimum threshold distance is reached. Once this threshold is reached the parking lot is assumed to be occupied and corresponding updates are made in the database. 
Module 2: Outgoing Vehicle - This is further sub-divided into three modules:

a). Sensor Actuation Module - In this module for the outgoing vehicles as the vehicle would move away from the sensor the distance detected by the Ultrasonic Distance Sensor keeps on increasing and when it increases beyond a certain fixed threshold the Parking Slot is assumed to be free. Status of the parking slots are updated in the database accordingly.

b). Parking Guidance Module - In this module, based on the updations from the above actuation module LEDs are lighted on the path from the freed slot to the exit of the parking in order to assist the Vehicle (driver) in exiting.

c). Image Processing - In image which is processed for drivers' face and number plate detection. This is followed by the matching process where the drivers' face and number plate detected here are matched with those taken at the time of entry. This is done to prevent incidents of theft etc. In case of mismatch signals are sent to the base station to call for human assistance.

\subsection{Detailed description}

As the car arrives the parking area, the system checks for vacant slot and an LED in front of the entry gate displays whether there is a vacant slot in the parking area or not. If there is a vacant slot, the Camera captures the image of the number plate of the car, before it enters the parking area; the second camera takes the image of the car driver from the driver's side. Both the images are sent to the computer placed at the entry gate to which these cameras are attached. Both the images are stored in the database for comparison at the time of exit.

\subsubsection{Face detection}

As the vehicle stands in the specified region, the driver is instructed to look towards the camera unit on the right side. The pressure sensors are deployed in the pre specified region at the entry gate to detect the presence of a vehicle in the region and send the message of presence of vehicle to the controller that activates the two camera units at the entry. One takes two images of the drivers' face. The other camera unit set at the lower front angle towards the vehicle takes the image of the number plate. These images are stored in the database. At the time of exit the images of drivers' face taken at the time of entry are matched with a new image taken at exit gate. The exit gate follows the same concept of specified region and pressure sensors as the entry gate discussed above. The images are matched for authentication using the LBP (Local Binary Pattern) operator and squared distance dissimilarity.

The local binary pattern (LBP) operator [14] is defined as a gray-scale invariant texture measure, derived from a general definition of texture in a local neighborhood. Due to its discriminative power and computational simplicity, the LBP texture operator has become a popular approach in various applications, including visual inspection, image retrieval, remote sensing, biomedical image analysis, motion analysis, environment modeling, and outdoor scene analysis.

Recent developments showed that the local binary pattern texture method also provides outstanding results in representing and analyzing faces in both still images and video sequences. LBP is a simple but very efficient texture operator which labels the pixels of an image by thresholding the $3 * 3$ neighborhood of each pixel with the value of the centre pixel and considers the result as a binary number. The value of the LBP code of a pixel $(\mathrm{x}, \mathrm{Yc})$ is given by:

$\mathrm{LBP}_{\mathrm{P}, \mathrm{R}}=\sum_{p=0}^{p=\mathrm{s}} s(G p-G c) 2^{p}$

Where Gc corresponds to the gray value of the centre pixel (Xc, Yc), Gp refers to gray values of $\mathrm{P}$ equally spaced pixels on a circle of radius $\mathrm{R}$, and $\mathrm{s}$ defines a thresholding function as follows:

$$
s(x)=\left\{\begin{array}{l}
1, \text { if } x \geq 0 \\
0, \text { otherwise }
\end{array}\right.
$$

The calculation of the LBP codes can be easily done in single scan through the image. The 256-bin histogram of the labels computed over a region can be used as a texture descriptor. LBPP, R encodes simple binary microstructures into a P-bit number. 


\subsubsection{Number plate recognition}

As the car reaches the parking entry gate, the cameras attached at the gate capture snapshots. One of the cameras captures the image of the number plate of the car. The number plate image is processed and the car number is extracted and stored in the database for comparison at the time of exit. This ensures security at the parking area. The process of number plate identification involves the main steps: Segmentation of characters (numerals and alphabets) and recognition of plate characters.

According to Kumar Parasuraman and P.Vasantha Kumar [15] for extracting the Plate region the algorithm uses edge detection algorithm and vertical projection method. In character segmentation step, filtering, thinning and noise removal are used. The algorithm is designed for real-time Indian license plate recognition and the input image is the image of the number plate taken from front of the vehicle by using the second camera placed at the entry gate. The camera is focused to capture the image of only the number plate to avoid complexities. The method comprises the following major steps, which are: filtration, binarization and smoothing process and noise removal which are depicted in Table 2.

After converting the image to binary, the next two steps remove any object near to the border of the image to get a clear picture. This is done by calculating the number of regions in the image, after the regions are calculated we calculate the area of each region, a threshold is predefined and the regions with area less than the threshold are considered to be extra noise and are removed. The regions with areas more than the threshold are retained in the final image. In this way, the number plate recognition final processed image is obtained and the resultant chaacter image is saved in the database.

Same process is repeated at the exit gate and the two final images are compared for authentication. The above mentioned algorithm is implemented in MATLAB and the Table 2 summarizes the steps with resultant images obtained. In this way the car number is extracted and saved to the database along with the driver's image to ensure security while he exits.

Since, the architecture and the demarcated slot for the car is fixed, the cameras at entry and exit are placed in such a way that exactly same images are captured. The positioning is done precisely so that camera angle and illumination at the entry and exit gate is absolutely identical. We then compare both the end result binary images pixel by pixel to perform the security process.

\subsubsection{Vacant Slot Identification}

Each slot in the parking area is given a slot number, corresponding to this slot number, an Arduino board pin is assigned for that slot. The status of the slot can either be 'VACANT' or 'OCCUPIED'. This status is stored in the system along with the slot number according to its occupancy and the directions from the entry gate to the slot. The Arduino pins are used to detect the changed status, initially the output at each vacant slot is 'LOW' that is a binary 0 . On being occupied, the status changes to 'HIGH' i.e. a binary 1.

As, a car arrives the car parking entry gate, the system is looked up for a 'VACANT' slot number, if a vacant slot is found, the slot number and directions are given to the user and he is directed to the particular slot as discussed in the next section. Identification of vacant slot in the parking area is done using the ultrasonic distance sensor mounted at the ceiling beam of each parking slot. The distance reading of each sensor is constantly monitored at the entry gate Arduino boards to which the sensors are connected via a wired connection. The flow graph for the module is presented in Figure 9.

A threshold is preset at the microcontroller and if the distance calculated by the ultrasonic sensor becomes less than the threshold, a signal is sent to the computer via wired communication between Arduino boards and the computer at the entry gate. The threshold is decided on the basis of height of beam and the placement of the sensor. As the car enters the particular parking slot designed for it, the car becomes the target for the ultrasonic sensor for distance calculation; it keeps on calculating its distance from the ceiling beam.

As the car is parked and the threshold is reached, the Arduino board's respective pin turns 'HIGH' indicating that the particular slot is no more vacant. The signal is transferred to the computer via serial communication and the database is updated, the slot number is no more taken to be vacant and its status flag is changed to be 'OCCUPIED'. The total number of vacant and free slots is also edited in the database accordingly. The signal sent to the computer is a 2 bit numeric signal, the first bit representing the designated serial number and the second representing the status of the slot i.e. ' 0 ' or ' 1 '. The flow graph for the module is presented in Fig 3. 

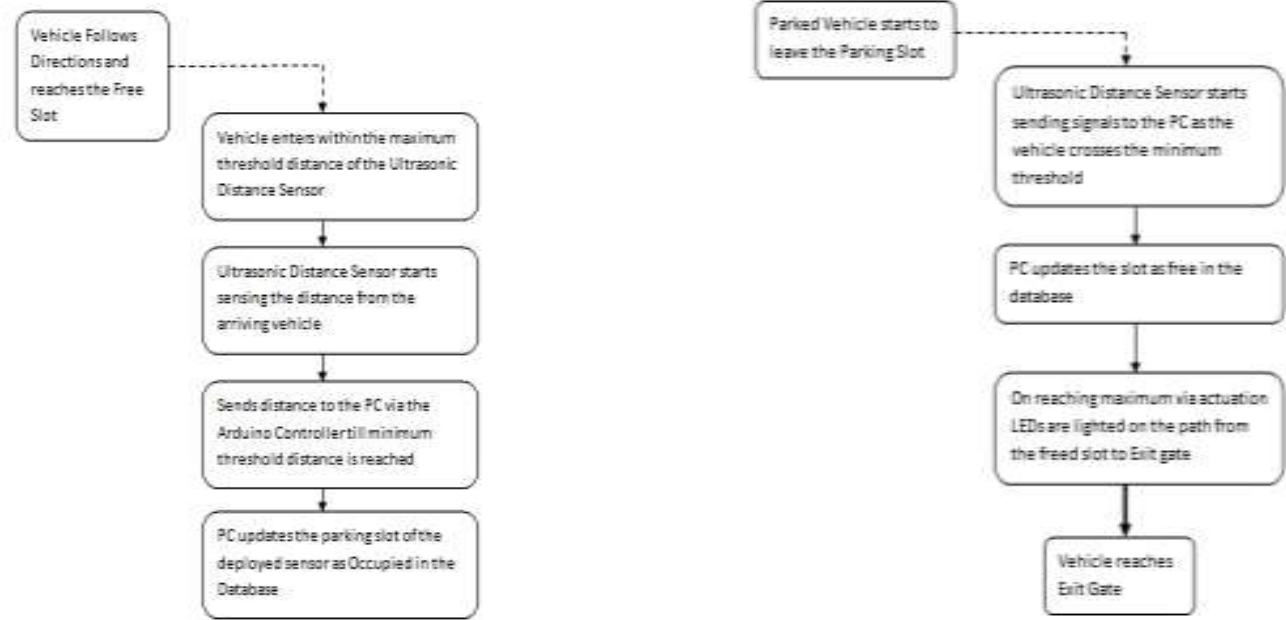

Fig. 3: Flow diagrams for Vacant slot Identification Module

\subsubsection{Automatic Guidance}

After the images are taken at the entry gate, the user is provided with the assigned slot number and directions to it from the entry gate. The file also contains the directions from the slot to the exit gate. The computer placed at the entry gate automatically generates a printed receipt of the file which the user can collect before he enters the parking area. The relevance and utility of the printout lies is that on returning back, the user is not supposed to memorize the slot number or the directions to get to the vehicle. He just has to follow the directions written on the receipt. This saves time and increases reliability, user ease and efficiency of the system.

In addition to the printed direction guidance, a voice enabled automatic direction based path to the designated slot is announced using the speaker attached at the entry gate. This reduces the inconvenience for the user and also proves to be helpful in case a person not literate enough to read is driving the vehicle. This voice enabled announcement uses Text-to-Speech synthetic voice software to perform the desired action and reads out the file generated by the computer. For the text-to-speech operation is performed using VOICEBOX [16] which is a speech processing toolbox consists of MATLAB routines that works with MATLAB version V6.5 and higher.

\section{RESULTS AND ANALYSIS}

Following are the results of the implementation of the modules of the system. The first module is the face detection and matching at the time of exit. Table1 shows the results of this module, it presents facial images taken at entry and exit, their histograms and the results of authentication (based on squared distance dissimilarity).

Table 1: Results of Face Detection Module

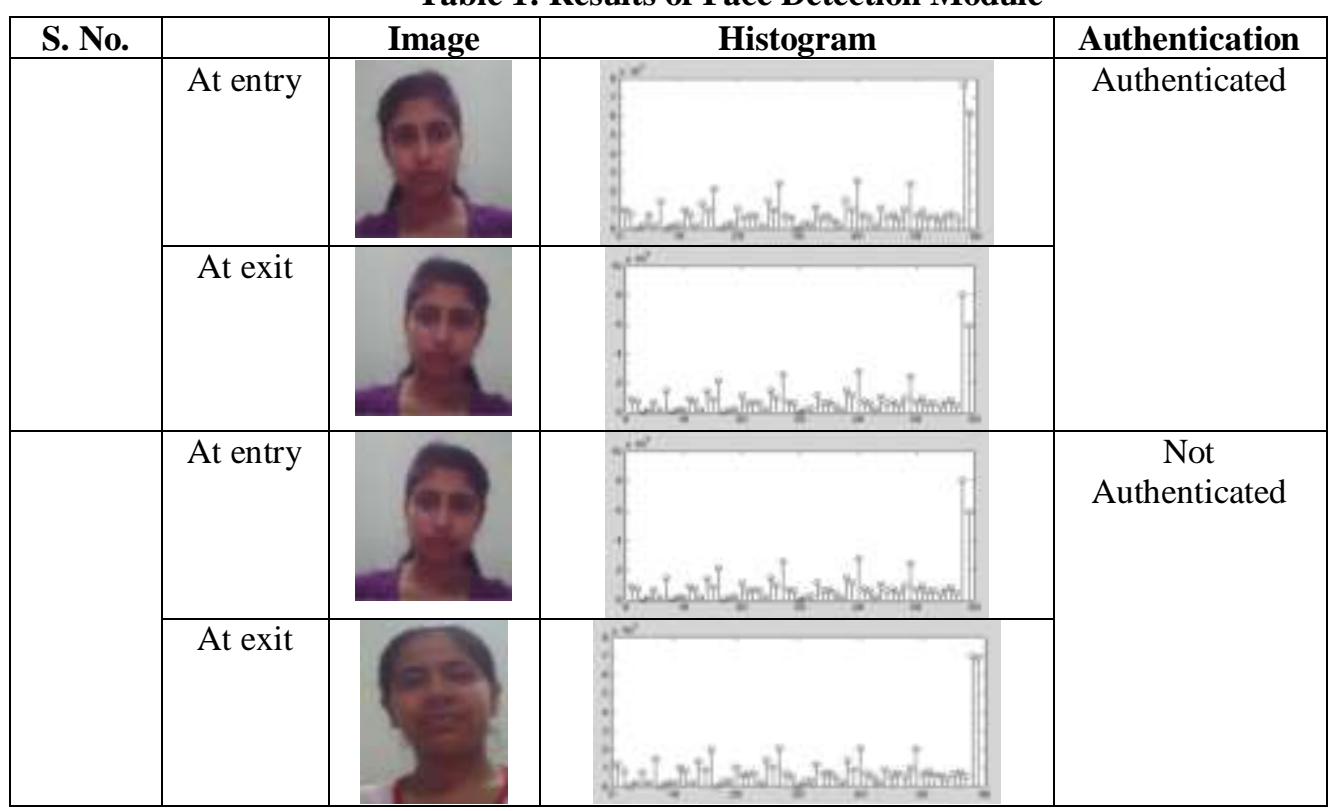


The results of next module are presented in table2. Figure shows the image of the number plate captured and the table presents further processing.

\begin{tabular}{|c|c|c|}
\hline Stages & Description & Resultant Image \\
\hline Filtering & $\begin{array}{l}\text { Apply low frequency filter } \\
\text { with convolution matrix. }\end{array}$ & $\frac{\text { CHO1AN0001 }}{n}$ \\
\hline Binarization & $\begin{array}{l}\text { By using MATLAB } \\
\text { functions applied in the } \\
\text { code }\end{array}$ & 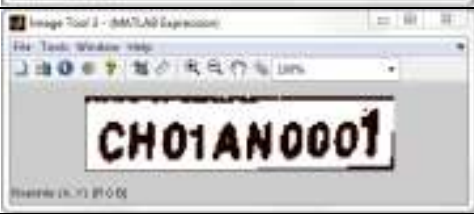 \\
\hline $\begin{array}{l}\text { Thinning using } \\
\text { contours }\end{array}$ & $\begin{array}{l}\text { To reduce the width of } \\
\text { characters, we also use a } \\
\text { thresholding index. }\end{array}$ & 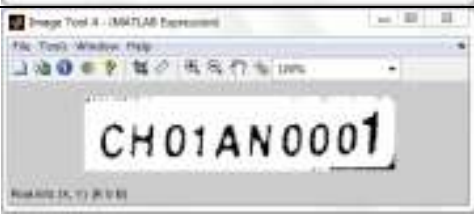 \\
\hline Noise Removal & $\begin{array}{l}\text { Remove extra noise and } \\
\text { small portions by using the } \\
\text { region properties like area } \\
\text { and setting a threshold } \\
\text { value, if more than the } \\
\text { threshold then retained. }\end{array}$ & 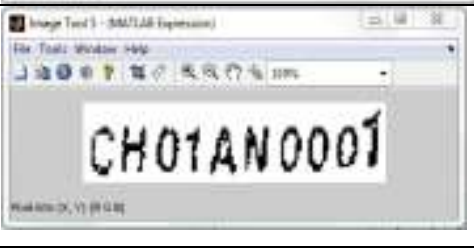 \\
\hline
\end{tabular}

Table 2: Results of Number Plate Recognition Module

Implementation of third module is done with the help of Ultrasonic Distance Sensors which sense the distance and send it Arduino controller from where it is forwarded for database updates to the PC via serial communication. Fig. $4 \mathrm{a}$ presents the view of prototype of this module. Fig. $4 \mathrm{~b}$ presents the Ultrasonic Distance values as the vehicle parks in and out of the slot. Fig. 5a and 5b: Plot of Ultrasonic distance values for CAR IN and CAR OUT

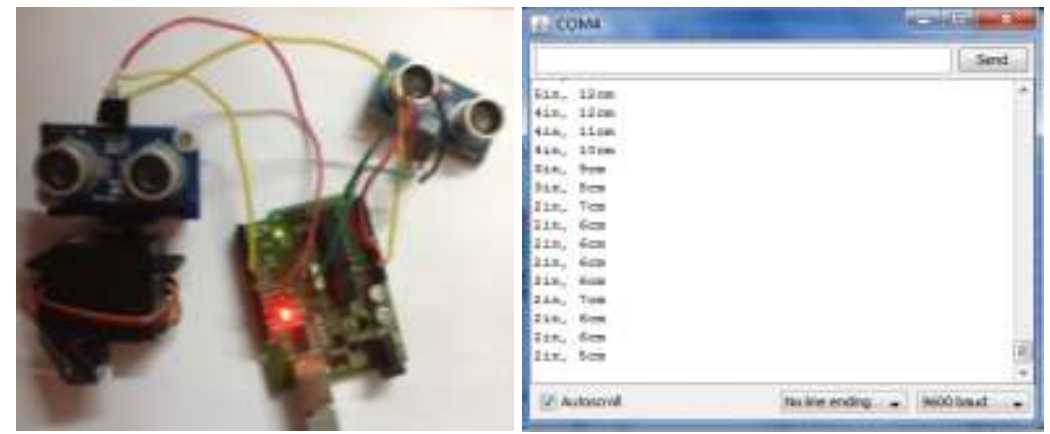

Fig. 4: Circuitry showing Ultrasonic Distance Sensor and Arduino b: Ultrasonic Distance values

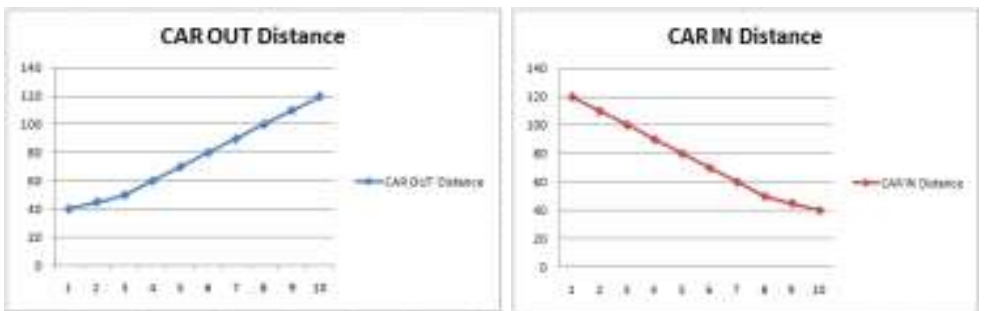

Fig. 5: Plot of Ultrasonic distance values for CAR IN and CAR OUT

Next module is the automatic guidance module. Fig. 6 presents the view of guidance provided for incoming vehicles and figure presents the guidance view for the outgoing vehicles. 

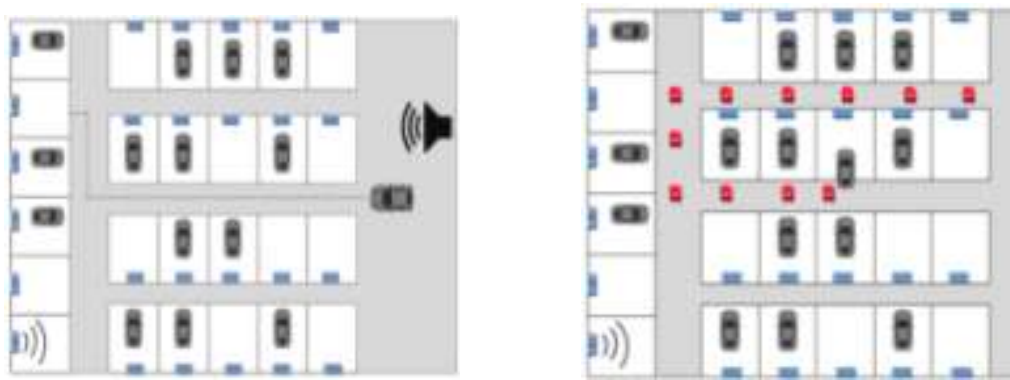

Fig. 6: View of guidance provided for a: incoming vehicles b: outgoing vehicles

VI. SYSTEM EVALUATION

The presented system is evaluated against the conventional parking systems for time and human effort it saves. In a conventional parking system the incoming vehicle has no information about any free parking slot available in the parking area. It searches for free parking slot moving in different directions in order to reach a free slot, this results in a longer searching time in cases where the parking area is highly occupied or the parking area itself is very large. The presented system AUTOPARK helps in reducing this searching time and effort by providing information as well as direction guidance towards the free parking slot. Fig. 7a and $7 \mathrm{~b}$ depict a logical and generalized view of how the searching time would change for conventional parking systems and AUTOPARK as the size of parking area increases and as the number of occupied slots in the parking area increases respectively.
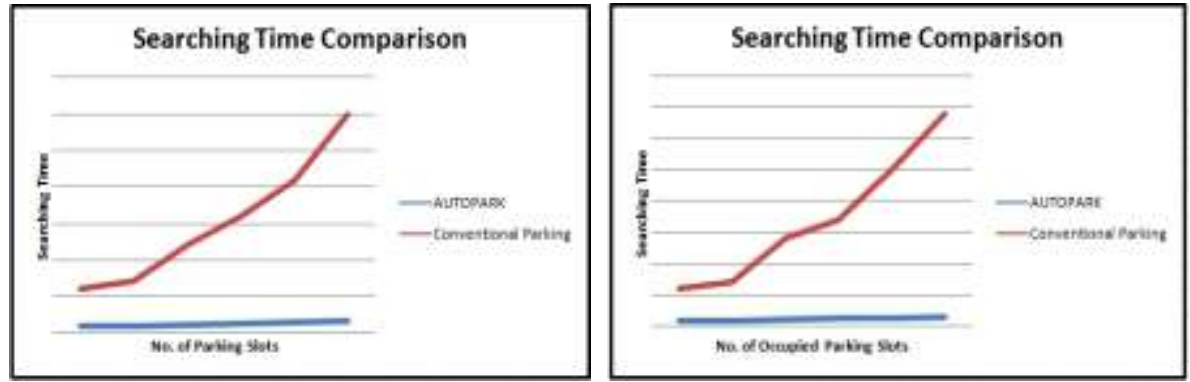

Fig. 7: Comparison with Conventional Parking Systems

As compared to other automated parking management systems most of them provide for the count of vehicles in the parking area or for LCD based display of number of vacant slots as presented in related work in Section 2. Whereas our AUTOPARK provides not only the number of free slots but also the specifications like which slot is free and automated voice guidance towards that slot. Even at the time of exit the vehicles are assisted with the help of LEDs towards the Exit gate. AUTOPARK also provides for security from theft via facial and number plate image processing and matching. Human intervention is also provided for via actuation in case of mismatch.

\section{CONCLUSION}

In this paper we presented an automated parking guidance and security system, based on an infrastructure based wired sensor actuator network. The system provides for automated voice guidance that directs an incoming vehicle towards a free parking slot and an LED based path guidance for outgoing vehicles from parking slots towards the exit. This results in efficient utilization of the parking area and saves a lot of human effort in searching and reaching a free parking slot. Along with the guidance the proposed system also provides for safety provisions for the vehicles in the parking area using image processing applications of face detection and vehicle number plate recognition. Images processed at the time of entry and exit are matched to authenticate the vehicle and driver thus providing safety, in case of mismatch human assistance is provided via actuation. Hence the proposed model is an efficient, economic parking system automation that provides for direction guidance and security saving both time and human effort. 


\section{REFERENCES}

[1] Padam, Sudarsanam, Singh Sanjay K., Urbanization and Urban Transport in India: The Sketch for a Policy.

[2] Abboud W.N., Automation of the Parking Industry: A Strategic and Managerial Overview, Massachusetts Institute of Technology, 1994

[3] Sayeeraman, Aswin, Ramesh, P.S., ZIGBEE AND GSM BASED SECURE VEHICLE PARKING MANAGEMENT AND RESERVATION SYSTEM, Journal of Theoretical and Applied Information Technology, 31st March 2012. Vol. 37 No.2

[4] Sangwon Lee, Dukhee Yoon and Amitabha Ghosh,: Intelligent Parking Lot Application Using Wireless Sensor Networks. Proceedings of IEEE conference, 978-1-4244-2249-4/08

[5] Jatuporn Chinrungrueng, Udomporn Sunantachaikul, Satien Triamlumlerd, Smart Parking: an Application of optical Wireless Sensor Network, Proceedings of the 2007 International Symposium on Applications and the Internet Workshops (SAINTW'07)

[6] Barton, J., J. Buckley, B. O’Flynn, S.C. O’Mathuna, J.P. Benson, T.O’Donovan, U. Roedig, and C. Sreenan, The D-Systems Project - Wireless Sensor Networks for Car- Park Management, Proceedings of the 65th IEEE Vehicular Technology Conference, Dublin, Ireland, Apr 2007, pp.170-173.

[7] IrisNet: Internet-scale Resource-Intensive Sensor Network Service, http://www.intel-iris.net

[8] Hobby, U.C., Visualizing sensor data with Arduino processing, http://www.uchobby.com/index.php/2009/03/08/visualizing-sensorwith-arduino-and-processing/.

[9] XBEE Tutorial, https://sites.google.com/site/xbeetutorial/example

[10] Manual by Martin Hebel and George Bricker, Getting Started with XBee RF Modules: A Tutorial for BASIC Stamp and Propeller Microcontrollers, Version 1.0, ISBN 9781928982562

[11] Ultrasonic Distance Sensor, www.parallax.com

[12] T.L. Floyd, Digital Fundamentals, 8th ed.,2009, pp. 288-340.

[13] Pressure Sensor, www.alldatasheet.com/MPXV5050

[14] Zhiguang Yang, Haizhou Ai, Demographic Classification with Local Binary Patterns, Department of Computer Science and Technology, Tsinghua University, Beijing 100084, China ahz@mail.tsinghua.edu.cn

[15] Kumar Parasuraman, Kumar P.Vasantha, An Efficient Method for Indian Vehicle License Plate Extraction and Character Segmentation, IEEE International Conference on Computational Intelligence and Computing Research 2010

[16] VoiceBox MATLAB, http://www.voicebox.com/ 